\title{
Comprensión de la derivada apoyada en la ingeniería didáctica como método de investigación
}

\author{
Understanding the derivative supported by didactic engineering as a \\ research method
}

\author{
Dilán Fabián Saraza-Sosa ${ }^{\mathrm{a}^{*}}$, Raúl Prada-Núñez ${ }^{\mathrm{b}}$, Charen Lisset Vera-Gutiérrez ${ }^{\mathrm{c}}$ \\ a* Semillero de Investigación en Educación Matemática, Licenciatura en Matemáticas, dilansaraza1@gmail.com, ORCID 0000-0001-7469-9748, \\ Universidad Francisco de Paula Santander, Cúcuta, Colombia. \\ ${ }^{b}$ Magister en Educación Matemática, raulprada@ufps.edu.co, ORCID 0000-0001-6145-1786, Universidad Francisco de Paula Santander, Cúcuta, \\ Colombia. \\ ${ }^{\mathrm{c}}$ Semillero de Investigación en Educación Matemática, Licenciatura en Matemáticas, charenl.vg@gmail.com, ORCID 0000-0002-6400-0275, \\ Universidad Francisco de Paula Santander, Cúcuta, Colombia
}

Forma de citar: Saraza-Sosa, D. F, Prada-Núñez, R. \& Vera-Gutiérrez, C. L. Comprensión de la derivada apoyada en la ingeniería didáctica como método de investigación. Eco Matemático, 10 (1), 42-56

Recibido: 1 septiembre 2018

Aceptado: 17 noviembre 2018

\section{Palabras clave \\ Ingeniería didáctica, derivación, comprensión gráfica de la derivada.}

\begin{abstract}
Resumen: El cálculo diferencial ha tenido diversas dificultades en el entendimiento de sus diversos conceptos, en especial en el tema de la derivada, donde se ha registrado que los estudiantes desarrollan una asimilación de tipo algorítmico y mecánico en la aplicación de sus reglas de derivación, despreciando la construcción gráfica y visual, la cual es importante para un desarrollo óptimo del saber mencionado. Por lo anterior se implementó la metodología de ingeniería didáctica en el tema de la derivada, con el fin de sentar bases de tipo epistémico y documental para conocer cuáles son las situaciones que generalmente se presenta entorno a la enseñanza de este saber, en base a lo anterior se aplicó una prueba de conocimiento para identificar el grado de comprensión adquirido por los docentes en formación de programa académico de Licenciatura en Matemática de la Universidad Francisco de Paula Santander, a fin de establecer lineamiento para la estructuración de unas secuencias didácticas que fomento un aprendizaje óptimo de la derivada
\end{abstract}

*Autor para correspondencia dilansaraza1@gmail.com

http://dx.doi.org/10.1016/j.eq/

2590-9215 C 2017 Universidad Francisco de Paula Santander. Este es un artículo bajo la licencia CCBYNCND 


\section{Keywords}

Didactic engineering, derivation, graphic understanding of the derivative

\section{Introducción}

En ocasiones se han presentado dificultades en la comprensión diversos conceptos relacionados a las matemáticas universitarias, especialmente en la asignatura de Cálculo Diferencial tal como se muestra en la investigación de Hernández-Suarez, Prada-Núñez \& Ramírez-Leal (2017), ocasionado por una mala orientación por parte de la enseñanza que indirectamente promueve el dominio de procesos algebraicos, restringiendo la comprensión y por ende llevando una mala formación en el aprendizaje y la apropiación de las temáticas estudiadas (Retana, 2013); (Pinto y Parraguez, 2015); (Gallo, Manrique y Prada, 2017). Tall (1993) expone que existen falencias en el aprendizaje del cálculo en cuanto a su representación visual, habiendo preferencia la manipulación numérica y simbólica; sin relacionarlo a la resolución de problemas.

En esta perspectiva Hitt (2003) sostiene la necesidad de la visualización matemática conectándola con diferentes representaciones en el aprendizaje del cálculo ya que al realizar manipulaciones meramente algebraicas se aborda ineficientemente la comprensión, limitando el significado de las nociones matemáticas que se buscan desarrollar. Lo anterior se relaciona a lo afirmado por Duval (2006)

... solo en matemáticas donde se requiere un amplio y complejo juego de transformación de representaciones para pensar y también porque un enfoque dualista de la actividad matemática conduce a negar su importancia cognitiva. De hecho, la comprensión conceptual surge de la coordinación de los diversos sistemas semióticos usados, y darse cuenta de la forma específica de representar para cada sistema semiótico es condición cognitiva para la comprensión. (p.166)

Dentro de ese orden de ideas la derivada es un tema impartido al final la asignatura de cálculo diferencial que no es ajena a la anterior premisa, presentando dificultades en la intelección de los conceptos relacionados a esta, siendo un indicador susceptible de investigación que ha encaminado al desarrollo diferentes trabajos enfocados a identificar y potenciar una mejor comprensión de la derivada 
de una función. Park (2013) después de orientar una unidad en la formación en este concepto, identificó las concepciones presentadas por los estudiantes, determinando que no se construían en su totalidad, construyendo ideas diferentes de la derivada de una función general y particular (teniendo una mejor asimilación en esta), sin relacionar las nociones de derivada y pendiente. Ruiz, Gutiérrez, y Garay (2018) con el objetivo visualizar escenarios de conceptos adjuntos a la derivada por parte de los educandos, se impartieron una metodología de enseñanza mediante el uso de dispositivos móviles, obteniendo un avance significativo en cuanto a la conceptualización de razón de cambio, advirtiendo también que prima la asimilación el concepto de derivada como la resolución operativa de ejercicios sin relacionarlos a su respectivo entendimiento geométrico utilizando la noción de limite, similar a las evidencias obtenidas por Briceño, Hernández y Espino (2018) donde después de aplicar un instrumento de tipo geométrico-variaciónal relacionado a la temática de la derivada y enfocado a determinar las ideas existentes por parte de los estudiantes, se obtuvieron tendencias hacia una asimilación algebraica relacionada con la fórmula del límite y las reglas de derivación, despreciando su respectiva comprensión gráfica, teniendo también nociones no complementarias de la tangente; como recta que solo toca la función en un solo punto, Estas afirmaciones resultan ser coincidentes con algunas conclusiones provenientes de trabajos realizados anteriormente por (Vrancken, Engler, y Müller, 2010), (Rodríguez, 2015), (Gutiérrez, Buitrago y Ariza , 2017), (Saraza y Prada, 2017).

Partiendo de lo anterior es necesario traer a consideración la importancia de fomentar una buena aprehensión de esta temática desde una perspectiva grafica como lo expone Weigand (2014) que plantea que es esencial fomentar un buen entendimiento del concepto de derivada que trascienda las reglas sintácticas de derivación y así crear bases fuertes para la continuación de la línea del cálculo y su articulación situación con otras disciplinas. Esta premisa encabeza el propósito de este trabajo de investigativo, que adopta la metodología de ingeniería didáctica (explicada seguidamente), con el fin fomentar una comprensión de conceptos de la derivada desde una perspectiva gráfica, sin descuidar su correspondiente asimilación algebraica.

\section{Materiales y métodos}

La metodología de investigación es la denominada ingeniería didáctica, Según Calderón y Corredor (2012) esta presenta una correlación entre un análisis epistémico y la implementación de un diseño didáctico. Los autores afirman que la particularidad esencial de este método de investigación es análisis dado durante el diseño las actividades (a priori) y el análisis posterior a su respectiva aplicación (a posteriori). Artigue (1995) la caracteriza como un método de "realizaciones didácticas" que conlleva la construcción y ejecución de secuencias de enseñanza, para posteriormente analizar los resultados encontrados, estableciendo una serie de fases a seguir en el respectivo proceso.

1) Fase 1 Análisis preliminar

2) Fase 2 Concepción y análisis a priori de las situaciones didácticas de la ingeniería

3) Fase 3 Experimentación

4) Fase 4 Análisis a posteriori y evaluación

En ese sentido la presente investigación está encaminada a agotar cada una de las fases en metodología de ingeniería didáctica, presentando en este documento avances de las primeras tres, donde se realizó en primer lugar un análisis epistémico del desarrollo evolutivo del concepto de derivada, en segundo lugar un análisis a priori, a partir de un rastreo de tipo documental derivado de un estado del arte alrededor de la comprensión del concepto de derivada, enfocado a la identificación de los aportes realizados previamente por otros investigadores en función de las falencias o potencialidades que continuamente se presentan en el momento de orientar esta temática. Y finalmente en base al análisis epistemológico y el análisis a priori se estructuro y aplicó un instrumento que identificara 
las dificultades de aprendizaje en los conceptos adjuntos a la derivada. La población sujeta a este trabajo fueron 32 docentes en formación, tomando una muestra de 5 estudiantes que cursaban la asignatura de Calculo Diferencia, en el programa académico de licenciatura en matemáticas de la Universidad Francisco de Paula Santander (UFPS) durante el segundo semestre del año 2018.

\section{Resultados y análisis}

\section{Análisis preliminar}

La transformación del concepto de derivada, tuvo un proceso evolutivo en el cual fue promovido por diferentes conceptos asociados, inicialmente con el problema del trazado de tangentes iniciada en la antigua Grecia con problemas de tipo geométrico, y seguidamente la determinación de máximos y mínimos, enunciada formalmente por Fermat, posteriormente Newton y Leibniz quienes llevan hasta su umbral el cálculo infinitesimal y por ende el concepto de derivada. Partiendo de lo anterior se presenta una descripción de las diversas etapas clave para la formación del concepto de derivada a partir de los trabajos de (Pino, Godino y Moll, 2011); (Vázquez y del Rincón, 1998) y (Pinto y Parraguez, 2015). Ver tabla I

Tabla 1. Origen epistemológico del concepto de derivada

\begin{tabular}{|c|c|}
\hline $320-260 \mathrm{AC}$ & $\begin{array}{l}\text { Los matemáticos griegos fueron por primeros en considerar las primeras nociones anteriores } \\
\text { al concepto de derivada, con el trazado de tangente expuesto en el libro III de los elementos } \\
\text { de Euclides; proposición XVII, que plantea la situación de trazar una recta tangente en } \\
\text { determinado punto de una curva (circulo), ya que en la matemática griega se desconocía un } \\
\text { método geométrico estándar para el trazado de tangentes, lo cual llevaba a considerarlas en } \\
\text { casos particulares. En esta proposición se involucraban conceptos de circulo, rectas } \\
\text { perpendiculares, radio, segmento de rectar, igualdad de ángulos y tangente. Euclides enuncia } \\
\text { la recta tangente a un círculo como "si una recta es tangente a un círculos y se traza el radio } \\
\text { en el punto de contacto, este radio es perpendicular a la tangente" Vera (1970) citado en ( ). } \\
\text { Así mismo en los libros de Apolonio de Pergamo, se puede visualizar un estudio de las } \\
\text { tangentes y determinación máximos y mínimos en sesiones cónicas. }\end{array}$ \\
\hline Siglo XIV & $\begin{array}{l}\text { Hacia la edad media se consideraron estudios de variación mediante apartados geométrico- } \\
\text { descriptivos, enfocados al entendimiento de problemas físicos, relacionados al movimiento } \\
\text { en general y particular se un objeto gracias a Merton College y Oresme, en la denominada } \\
\text { regla de Merton que involucraba el grafico de la distancia recorrida a una aceleración } \\
\text { uniforme. Los procedimientos desarrollados eran principalmente por la representación de } \\
\text { casos particulares, las que eran sometidas al análisis de situaciones representadas } \\
\text { geométricamente y presentando procedimientos argumentativos o visuales ya que no se tenían } \\
\text { métodos de generare para problemas de variación, desarrollando intuiciones prematuras de la } \\
\text { noción limite. }\end{array}$ \\
\hline Siglo XVI & $\begin{array}{l}\text { Los trabajos de Oresme sobre el movimiento condujeron a Galileo a estudiar la composición } \\
\text { vectorial del movimiento y describir la trayectoria de un proyectil representando su } \\
\text { movimiento a partir de un gráfico donde se relaciona el desplazamiento y tiempo, instruyendo } \\
\text { ideas de trazado de tangente a distintas curvas. Asi mediante la argumentación cinemática se } \\
\text { afianzan conceptos de la tangente en términos de la composición de dos movimientos. Estos }\end{array}$ \\
\hline
\end{tabular}


métodos de trazado de tangentes eran generalizados y extensivos sin considerar la parte infinitesimal, evitando la determinación de todas las velocidades.

En este punto se articula el álgebra al estudio de problemas relacionados a la geometría y ecuaciones de sistemas de coordenadas, dejando a un lado el estudio geométrico-descriptivo. Descartes, por ejemplo, planteaba problemas geométricos como el de la tangente en expresiones algebraicas, como su método de círculos geométricos, la dificultad ante el análisis y solución de problemáticas a partir de ecuaciones algebraica y geometría analítica es que se volvía tedioso y extensivo al expresar algunas curvas en términos algebraicos debido a los grandes cálculos. En esta época muchos matemáticos trataban de dad una solución general al a la noción de tangente.

Fermat y Barrow desarrollaron métodos en la determinación de la tangente y máximos y mínimos. Analizando la proximidad infinitesimal de la secante hacia la tangente, construyendo así las primeras ideas intuitivas de límites, derivadas a partir de apartados

Siglo XVII algebraicos, geométricos e infinitesimales.

Newton y Leibniz se les atribuye la invención del cálculo infinitesimal, con algunas consideraciones en sus métodos, por parte de Newton genero algoritmos para la determinación de máximos y mínimos, tangentes y curvaturas, realizando una comprensión con sentido infinitesimal, igual a la presentada por Barrow y Fermat llevando la hacía una conceptualización mecánica, relacionada al movimiento continuo, denominado configuración de fluxiones, siendo las variables $\mathrm{x}$ e y los movimiento de las fluxiones lo que comúnmente se conoce como la derivada. En ese sentido Leibniz presento un interés por desarrollar una mejor claridad de los conceptos involucrados en la determinación de máximos, mínimos, tangente y puntos de inflexión, presentando lo que conocemos como calculo diferencial, y exponiendo algunas fórmulas de derivación.

A partir de esta época se empiezan a sentar bases para la formalización de conceptos relacionados al cálculo infinitesimal a partir de conceptos función, límites (siendo primordial Siglo XVIII para el entendimiento de la derivada) y derivadas desde una perspectiva más formal, en adelante predominando un carácter mayormente algebraico y en ocasiones articulándolo con el geométrico, gracias a las diferentes contribuciones de matemáticos como Euler, Cauchy, Lagrange, entro otros.

\section{Análisis a priori}

En el análisis a priori se han tenido en cuenta la revisión de tipo estado del arte, en diversos antecedentes que se han enfocado en la determinación de diversas dificultades o destrezas presentadas comúnmente por los estudiantes y/o docentes cuando se orienta la conceptualización de la derivada de una función. 
Tabla 2. Revisión de antecedentes

\begin{tabular}{ll}
\hline \multicolumn{1}{c}{ Autores } & \multicolumn{1}{c}{ Descripción } \\
\hline $\begin{array}{l}\text { Sánchez, García y } \\
\text { Llinares (2006) }\end{array}$ & $\begin{array}{l}\text { Los estudiantes presentan un desarrollo comprensivo del concepto de la derivada } \\
\text { cuando se articulan diferentes medios representativos, como el caso del uso gráfico y } \\
\text { carácter puntual } f^{\prime}(a) \text { y global } f^{\prime}(x) \text {, tanto en situaciones graficas como analíticas, en } \\
\text { ese sentido recomiendan la articulación de las dos en estas situaciones. }\end{array}$ \\
\hline Cervantes, Camarena y & $\begin{array}{l}\text { Presentan una propuesta didáctica en la enseñanza de la derivada desde una perspectiva } \\
\text { enfocada a la modelación de situaciones de contexto, con el objetivo de cambiar el } \\
\text { enfoque tradicional con contenidos abstractos impartido habitualmente. Concluyendo } \\
\text { que existe una mejor apreciación del concepto de derivada por parte de los estudiantes, } \\
\text { desde el enfoque de modelación al tradicional. }\end{array}$ \\
\hline
\end{tabular}

Observan tendencias por parte de algunos estudiantes a considerar la derivada como Amaya Rojas y Ballen un proceso algorítmico, sin relacionarlo a la razón de cambio o la pendiente de la recta (2009) tangente, así mismo son capaces de aplicar los criterios de primera y segunda derivada, pero no lo relacionan con la función derivada, también no existe una relaciona de gráfica de la función con la de la función derivada.

Ariza y Llinares (2009)

En la población estudiada se analizó el concepto de la derivada en la articulación de situaciones económicas, demostrando que existe una fuerte inclinación por la utilización del registro algebraico en comparación al manejo que desarrollaron los estudiantes frente al lenguaje gráfico, sustentando que los educandos que realizaban los dos lenguajes presentaban una mayor asimilación del concepto de derivada.

Aplicaron una prueba con el fin de indicar las concepciones presentadas por los estudiantes respecto al tema de la derivada, llegando a diversas conclusiones

Carabús (2009) relacionando la derivada como la que determina el crecimiento y decrecimiento en la función, un valor único de la pendiente de la recta tangente, como un algoritmo aplicado a las funciones sin significado, entre otros. Los autores advierten de forma general falencias en la concepción del pensamiento variacional y gráfico.

Vrancken, Engler y Müller (2010)
En la implementación de diversos medios de representación, los estudiantes presentan mayor facilidad al trabajar en los registros numéricos y dificultades en los lenguajes gráficos y algebraicos, en ese sentido concluyen que la propuesta presentada, que potencia el enfoque variacional promovió un mejor entendimiento de la idea del concepto de derivada.
Badillo, Azcárate y Font (2011)
Describe los niveles de comprensión entre las relaciones de $f^{\prime}(a)$ y $f^{\prime}(x)$ que poseen 5 profesores de matemáticas, determinando variabilidad en la asimilación grafica hasta la algebraica de los encuestados, exponiendo que algunos ambigūedades en la relación $f^{\prime}(a)$ y $f^{\prime}(x)$, dificultades en la relación de $f(x), f^{\prime}(x)$ y $f^{\prime}(a)$ y no argumentación en el proceso de determinación de derivada mediante regla o el límite.

Orhun (2012)

Determino errores conceptuales por parte de los estudiantes, argumentando que no se desarrolla un entendimiento del tema de la derivada, ya que son relacionadas a acciones operativas, sin ninguna interpretación grafica de la derivada de una función. El autor 
también postula dificultades en las conexiones entre la función original y la función derivada, atribuyendo a $f^{\prime}(x)$ ser la gráfica $f(x)$.

Alvarez, Colorado y Ospina (2013)

Vega, Carrillo y Soto (2014)

Vrancken y Engler (2014)

Hashemi, Kashefi y Rahimi (2014)

Cuevas, Rodríguez y González (2014)

Pino, Godino y Font (2015) dificultades en la solución de situaciones relacionadas al concepto de la derivada desde diferentes perspectivas, como la relación de la derivada con la razón instantánea de cambio, diferencia entre $f^{\prime}(x)$ y $f^{\prime}(a)$. Se exhibe una mejor asimilación al considerar la derivada como pendiente de la recta tangente.
Santoyo, Rangel, Teyes y Puga (2016)
Analizan la orientación del concepto de derivada en dos grupos, uno de control donde se implementó una metodología de enseñanza de tipo tradicional y el otro de tipo experimental donde se impartieron los saberes mediante unas estrategias didácticas enfocada al fortalecimiento de conceptos previos de carácter simbólico, conceptual y procedimental. Concluyendo que aun en los dos grupos se construyen ideas del concepto de derivada, pero el experimental logra un aprendizaje significativo de tipo conceptual y procedimental en la resolución de problemas.

Identificaron los aprendizajes desarrollados por los estudiantes en el concepto de derivada y sus respectivas aplicaciones, encontrando debilidades en la asimilación de este concepto en cuanto a derivada de una función general y particular en un punto dado y limitaciones en la asimilación desde una perspectiva visual y geométrica.

Implementaron una secuencia de aprendizaje enfocada a promover la asimilación conceptual de la derivada, estructurando situaciones de variación en contexto de situaciones físicas, como cálculo de razones de cambio y pendientes abordando diversos medios de representación como el verbal, grafico, numérico y analítico, realizando relaciones entre estos. Las dificultades presentadas por los estudiantes fueron de tipo conceptual y algorítmico relacionado a conocimientos previos necesarios.

Postulan resultados en los que muestran diversas dificultades en el aprendizaje de la derivación. A partir del instrumento aplicado se obtienen falencias frente a preguntas de tipo conceptual, respuestas enfocadas más al aspecto simbólico de la derivada que el entendimiento gráfico (limitado la interpretación geométrica del concepto), sin una respectiva relación de los dos.

Introducen el concepto de derivada de una función real mediante el uso de recursos digitales, en donde enfatizan el no utilizar un medio de representación, sino por el contrario articular diferentes medios representativos. En lo resultados los estudiantes al inicio presentaban falencias en conceptos aritmético, algebraicos y de funciones. A partir del uso de las herramientas tecnológicas se promovió el desarrollo intuitivo de la derivada de una función real.

Establecieron dos grupos uno con una metodología tradicional y en otro se utilizó una propuesta experimental, obteniendo en esta ultima una mejor apreciación del concepto de derivada de una función y la interpretación de diversos registros de representación, desarrollando así habilidades en la resolución de problemas de optimización. 
En el diseño y aplicación de un instrumento con el objetivo de clasificar el razonamiento geométrico del concepto de la derivada, se obtuvo que los estudiantes realizaban afirmaciones visuales de las gráficas, sin reconocer la representación geométrica de la derivada, desarrollo procesos infórmales en la determinación de las pendiente, a partir de ilustraciones de pendiente dada y solo una pequeña población entendian el proceso de hallar la pendiente de la recta tangente a la gráfica relacionado a la derivada de una función

Mendoza, Alemán y

Nieves (2017)
Caracterizan las dificultades que presentan los estudiantes en torno a la derivación de funciones reales, a partir del análisis y encuentras aplicadas. Se enuncia falencias de tipo cognitivo y procedimental en relación a comprensión de la derivada como razón de cambio, conceptualización de la derivada desde la definición de limite, la derivada en un punto específico, también dificultades de tipo algebraico y aritmético en la aplicación de las reglas de derivación, proponiendo así la implementación de un objeto virtual de aprendizaje (OVA) enfocado al aprendizaje de estos saberes.

\section{Experimentación}

En base al análisis epistémico donde se identificaron los conceptos claves en el desarrollo del concepto de derivada a nivel epistemológico y también a partir del análisis a priori de las diferentes investigaciones que han encontrado las falencias presentadas comúnmente en la construcción de este concepto se estableció una prueba de conocimientos enfocada a caracterizar las concepciones que presentan algunos estudiantes de Licenciatura en Matemáticas de la UFPS que cursaron la asignatura de Cálculo Diferencial durante el segundo semestre del 2018.

3.1 Prueba de conocimientos. Se construyeron 5 ejercicios establecidos a fin de identificar las falencias o potencialidades que poseen los estudiantes en los diferentes registros de representación (algebraico, numérico y gráfico) involucrados en la determinación de diversos conceptos relacionados a la derivada de una función. Partiendo de lo anterior se expondrá a nivel general el desempeño obtenido en la aplicación del instrumento (ver tabla 3) acompañado del objetivo que se planteó para la respectiva actividad.

\subsubsection{Presentación de resultados a nivel general}

Los estudiantes se nombraron con las letras de A, B, C, D y E. En los niveles de desempeño se caracterizaron cuantitativamente dependiendo de los puntajes obtenidos en cada una de las actividades, considerados de la siguiente forma, desempeño bajo $(0-0,25)$, desempeño regular $(0,26-0,51)$, desempeño medio $(0,52-0,77)$ y desempeño alto $(0,78-1)$.

Tabla 3. Resultados de la prueba de Conocimiento

\begin{tabular}{|c|c|c|c|c|c|}
\hline Estudiantes & Actividad $\mathrm{N}^{\circ} \mathrm{l}$ & Actividad $\mathrm{N}^{\circ} 2$ & Actividad $\mathrm{N}^{\circ} \mathbf{3}$ & Actividad $\mathrm{N}^{\circ} 4$ & Actividad $\mathrm{N}^{\circ} \mathbf{5}$ \\
\hline A & Regular & Regular & Bajo & Bajo & Alto \\
\hline B & Alto & Regular & Bajo & Bajo & Alto \\
\hline $\mathrm{C}$ & Alto & Medio & Bajo & Bajo & Alto \\
\hline D & Regular & Regular & Bajo & Bajo & Alto \\
\hline $\mathbf{E}$ & Regular & Regular & Bajo & Bajo & Alto \\
\hline
\end{tabular}




\subsubsection{Actividades en la prueba de conocimiento} aplicada.

Se identifica inicialmente el aprendizaje al solucionar de forma óptima el planteamiento que se presenta, para posteriormente comentar lo desarrollado por los estudiantes, teniendo en cuenta las dificultades y fortalezas presentadas.

El primer punto se enfocó a identificar si existía el pensamiento algebraico en la solución algorítmica de los ejercicios planteados, así mismo se evidenciaría la asimilación algorítmica de las reglas de derivación (regla del producto, del cociente, de la cadena, entre otras) al hallar las derivadas sucesivas (derivadas de orden superior) de las funciones expuestas.

1. Halle la derivada de orden superior que se le indica frente a cada función.
a. $f(x)=7 x^{4}+4 x^{3}-5 x^{2}+x \quad \frac{d^{3} y}{d x^{3}}$
b. $f(x)=\frac{x}{x+4}$ $f^{\prime \prime}(x)$
c. $\sqrt[3]{\left(x^{2}-1\right)^{2}}$
$f^{\prime \prime}(x)$
d. $f(x)=3 x^{2} \sqrt{x}+\frac{4}{\sqrt[3]{x}} \quad f^{\prime \prime \prime}(x)$

En la función polinómica presentada de primera no se concedió ninguna problemática; en la función racional del literal $\mathrm{b}$ el estudiante $\mathrm{D}$ la enunció sin realizarle algún proceso, como se muestra en la tabla de resultados, los estudiantes $\mathrm{B}$ y $\mathrm{C}$ solucionaron óptimamente los ejercicios planteados, contrario a las soluciones presentadas por A, D y E quienes tuvieron falencias de tipo algebraico en los dos últimos literales, en especial en el d donde el proceder en su solución fue inadecuado. Los estudiantes A, B, D, E no recuerdan o no saben cómo aplicar la regla de la cadena al literal c, y tres de estos la dejaron enunciada solamente, aunque no es un factor a considerar, ya que es de carácter memorístico, por lo menos uno se interesó en encontrar la solución por otro método.

Partiendo de lo anterior, se puede inferir que se presenta un nivel de solución algorítmica a los ejercicios expuestos por partes de los estudiantes, aunque se evidencias algunas falencias de tipo procedimental y algebraico.

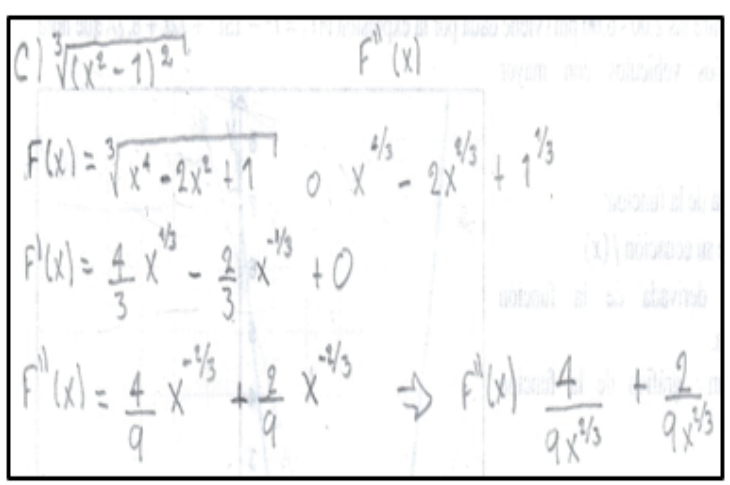

Figura 1. Resolución del estudiante B al literal c

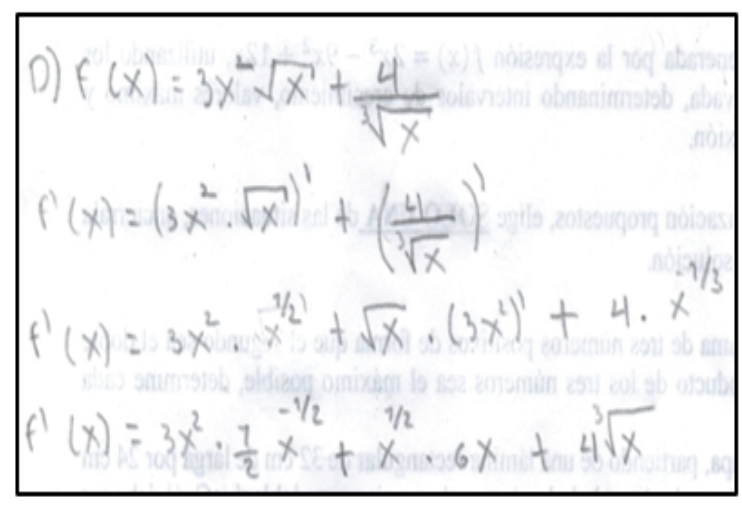

Figura 2. Resolución del estudiante A al literal d

La segunda actividad se estructuró para analizar la relación que existe en el registro gráfico entre la función $\mathrm{f}(\mathrm{x})$ y la función derivada $\mathrm{f}^{\prime}(\mathrm{x})$, distinguiendo las nociones en cuanto a la recta tangente en un punto cualquiera y en los distintos puntos respecto a la función dada. En ese sentido se evidenciará si es mayor la comprensión del registro gráfico de la derivada con respecto al registro algebraico, analizado en el ítem anterior.

2. Considere la función $y=x^{3}-1$. a) Haga un bosquejo de su gráfica tan cuidadosamente como pueda; b) Dibuje la recta tangente en $\mathrm{x}=2 \mathrm{y} \mathrm{x}=0$; $\mathrm{c}$ ) Estime la pendiente y la ecuación de la recta tangente en los valores de $\mathrm{x}$; d) grafique la respectiva función derivada $\mathrm{y}^{\prime}$ 
En este punto se evidenció un desempeño regular en el manejo del registro gráfico de la derivada. Todos graficaron la función dada normalmente, partiendo de ahí se pedía graficar la recta tangente en los puntos dados. Los estudiantes $\mathrm{A}, \mathrm{B}, \mathrm{D}, \mathrm{E}$ trazaron estas rectas cortando el recorrido de la función en el punto $\mathrm{x}=2$, es decir, una recta vertical en el punto dado, y ninguna en el punto cero; solo el estudiante $\mathrm{C}$ trazó bien las rectas tangentes. En cuanto a la de la ecuación de la recta, el estudiante B pudo establecer la relación entre la derivada y la pendiente de la recta tangente para la ecuación de esta misma. En este punto se evidencia una pobre concepción de la relación entre $\mathrm{f}(\mathrm{x}) \mathrm{y}$ $\mathrm{f}^{\prime}(\mathrm{x})$, teniendo nociones del registro algebraico a nivel procedimental pero no visual. Finalmente, se resalta la evidente falta de argumentación a las acciones realizadas.

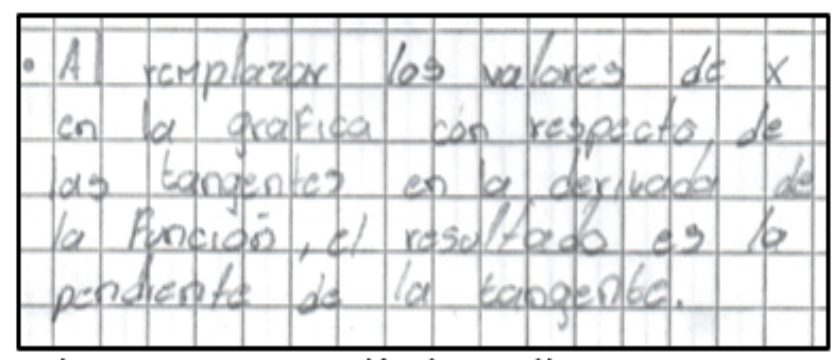

Figura 3. Argumentación de estudiante B

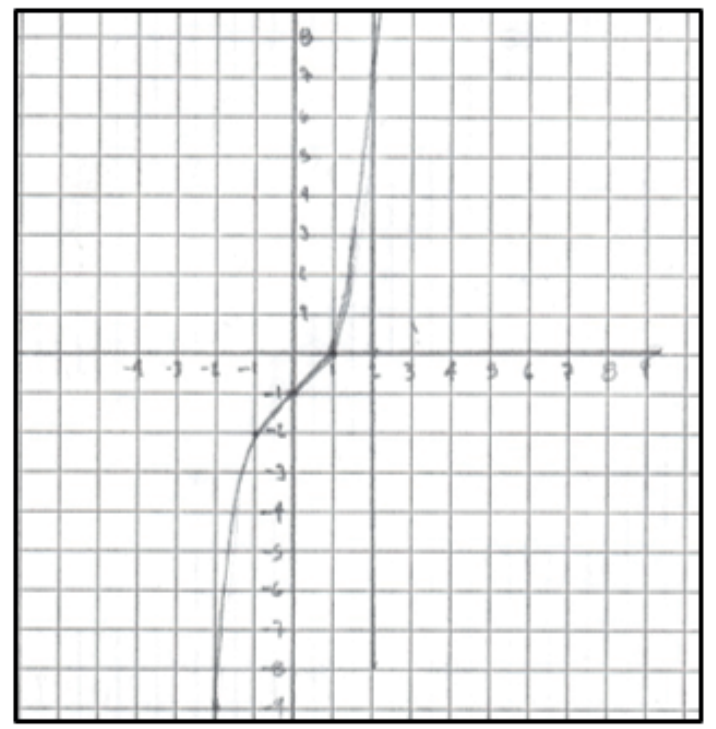

Figura 4. Gráfica presentada por el estudiante B

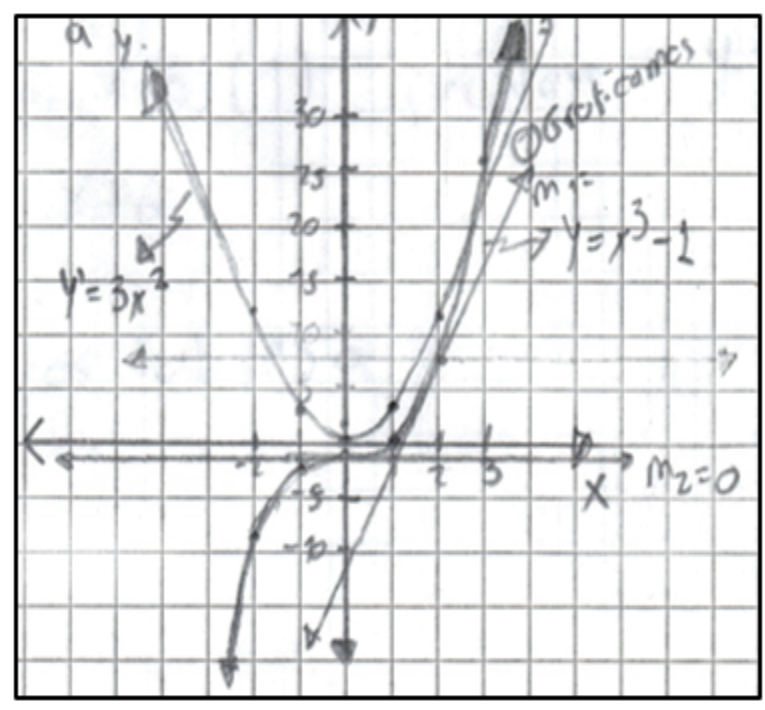

Figura 5. Grafica presentada por el estudiante C

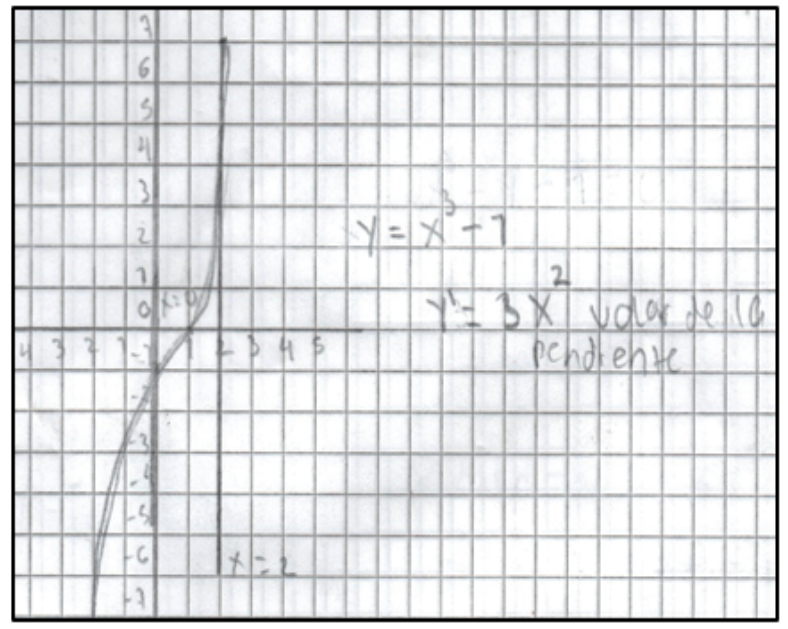

Figura 6. Grafica ilustrada por el estudiante A

La tercera y cuarta actividad se enfocó en los conceptos del criterio de la primera y segunda derivada, con el fin de identificar si el estudiante articula procedimientos algebraicos en la determinación de los puntos críticos (máximos y mínimos) respecto a la gráfica de la función que se les pide modelar. Así mismo, se pretendía determinar la forma como solucionan problemas de optimización de tipo estándar presentado comúnmente, en los cuales interfieren conocimiento de tipo procedimental en el análisis y la solución de las diversas situaciones. 
Realice el bosquejo de la gráfica generada por la expresión $\mathrm{f}(\mathrm{x})=2 \mathrm{x}^{3}-9 \mathrm{x}^{2}+12 \mathrm{x}$, utilizando los criterios de primera y segunda derivada, determinando intervalos de crecimiento, valores máximos y mínimo, concavidad y punto de inflexión.

De los siguientes ejercicios de optimización propuestos, elige solo una de las situaciones, enciérrala en un círculo e intenta determinar su solución.

Expresa el número 60 como la suma de tres números positivos de forma que el segundo sea el doble del primero. Se desea que el producto de los tres números sea el máximo posible, determine cada número.

Se desea construir una caja, sin tapa, partiendo de una lámina rectangular de $32 \mathrm{~cm}$ de larga por $24 \mathrm{~cm}$ de ancha. Para ello se recortará un cuadradito (de lado x) en cada esquina y se doblará. ¿Cuál debe ser el lado del cuadradito cortado para que el volumen de la caja resultante sea máximo?

Se ha observado que, en autopista norte de salida de Bogotá, la velocidad de los vehículos en horas de la tarde (entre las 2:00 - 6:00 pm) viene dada por la expresión $\mathrm{v}(\mathrm{t})=\mathrm{t}^{3}-15 \mathrm{t}^{2}+72 \mathrm{t}+8$. ¿A qué hora circulan los vehículos con mayor velocidad?

Estas dos actividades se presentan conjuntamente, ya que el desempeño en la resolución de estos puntos fue bajo, los estudiantes solo hallaban la primera o segunda deriva y las dejaban

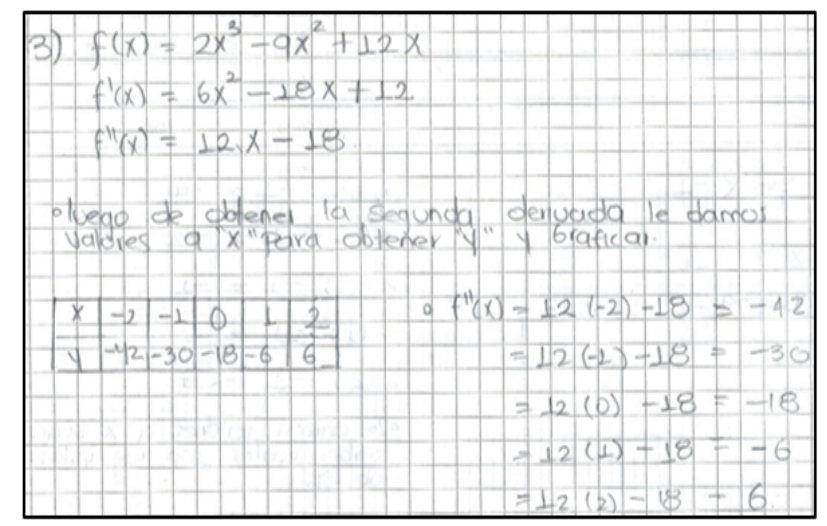

Figura 7. procedimiento realizado por el estudiante E enunciadas, trataban de formular analíticamente la situación sin relacionarlo a la derivada, o lo que es equivalente a afirmar que no procedían en la determinación de los valores máximos y mínimos o de punto de inflexión, lo que permite intuir que el tema no se hubiese llegado a desarrollar de forma profunda. A nivel general, no se evidencia ninguna relación del concepto de derivada con la construcción de la gráfica o problemas de tipo aplicado a situaciones de contexto.

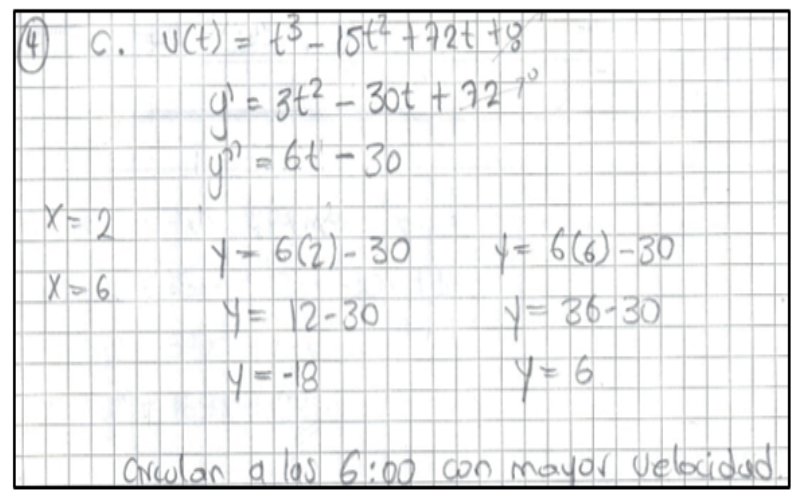

Figura 8. procedimiento realizado por el estudiante D

En el punto final se pretendía evidenciar la relación entre la gráfica de una función (en este caso visualizada y teniendo que determinar la ecuación que modelaba su comportamiento) y su respectiva derivada, la cual tenían que graficar después de encontrar la función derivada. Con este ítem se analiza como los estudiantes transitan desde el registro gráfico al registro algebraico, en el entendimiento de la noción de derivada.

Dada la gráfica de la función:

Determine su ecuación $\mathrm{f}(\mathrm{x})$

Halle la derivada de la función encontrada. Realice un gráfico de la función derivada. 


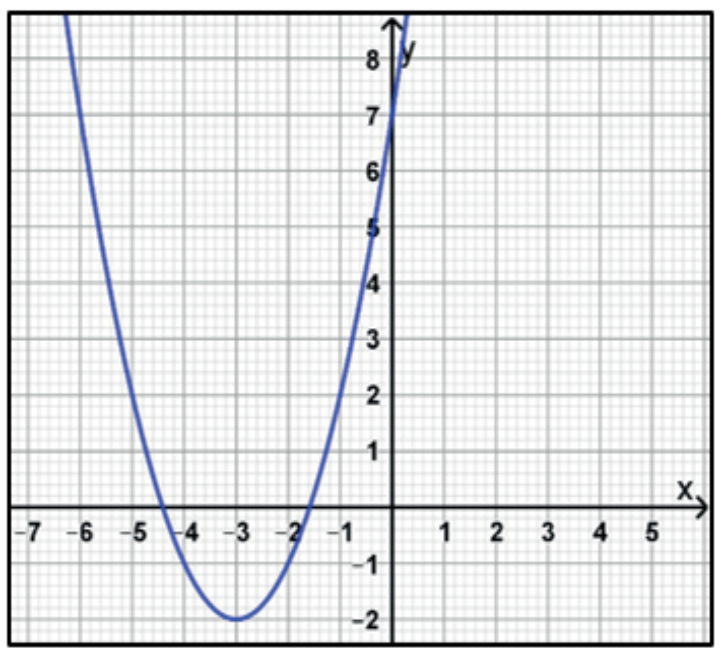

Figura 9. Grafica de la $\mathrm{f}(\mathrm{x})$ presentada

En este ítem fue dónde los estudiantes exhibieron mejor nivel de compresión, por ello todos tienen un desempeño alto en los resultados presentados. Todos los estudiantes determinaron la ecuación de la función a partir del análisis de la respectiva grafica presentada, identificando el vértice y punto de corte en el eje de las ordenadas, partiendo de esta identificación de valores, modelaron la función y hallaron su respectiva derivada, en comparación a la actividad del punto 2 se presentó una mejor relación entre estas dos funciones.

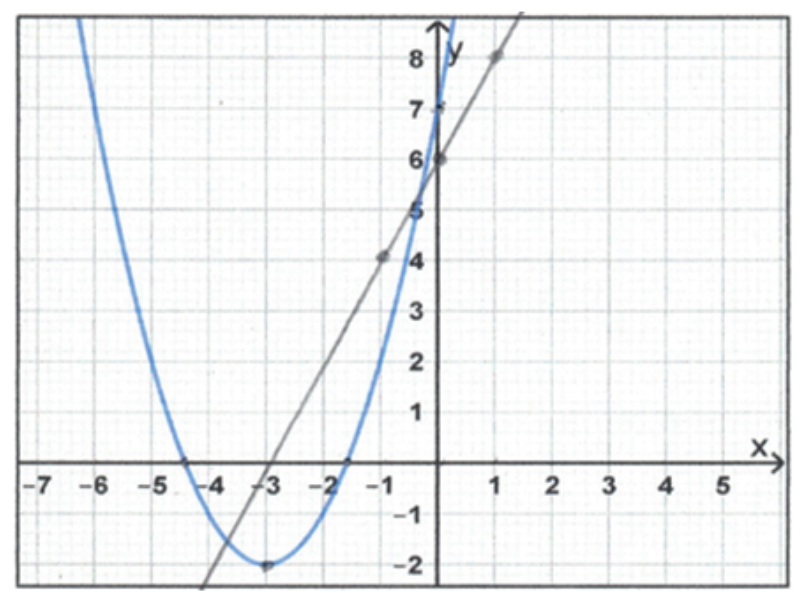

Figura 9. Grafica $\mathrm{f}^{\prime}(\mathrm{x})$ trazada por el estudiante $\mathrm{D}$

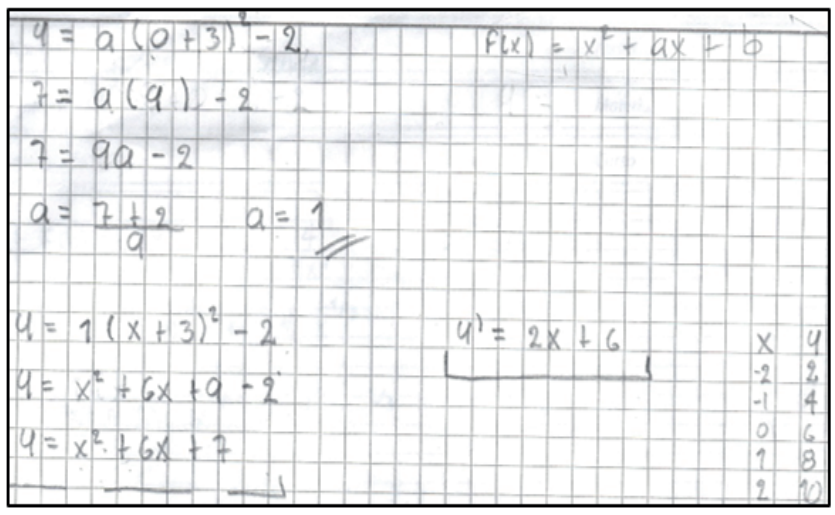

Figura 10. Proceso desarrollado por el estudiante B

\section{Conclusiones}

En base a lo encontrado en el análisis epistémico se evidencia que el concepto de derivada tuvo una evolución dese una perspectiva geométrica y visual, hasta su respectiva formalización en términos algebraicos, contrario a los hallazgos encontrados en el rastreo de tipo documental en el cual los estudiantes desarrollan una fuerte noción de la derivada de una función como el proceder algorítmico en la aplicación de las diferentes reglas, despreciando en gran medida el respectivo entendimiento gráfico. Lo anterior similar a lo encontrado en la prueba de conocimiento realizada en esta investigación, donde también se logró identificar falencias en la resolución de los puntos presentados. Asi en la aplicación de las actividades que articulaban el pensamiento gráfico- algebraico, se notan falta de conocimiento de tipo conceptual que les permitieran abordar lo pedido en esos puntos, un ejemplo es el mal trazado que dieron algunos estudiantes a la rectan tangente en los puntos dados y de igual forma los argumentos de las nociones que poseían, aunque se fueron mayores las dificultades en el punto 2 en el que se transitaba del pensamiento algebraico al gráfico, hubo mejor recepción cuando se presentó desde la parte visual de la gráfica a su ecuación acción que se debía hacer en el punto 5.

Las situaciones que tenían como fin identificar como utilizan la derivada en términos de aplicación a su respectivo concepto en la determinación de los puntos críticos o problemas de optimización 
fueron en las que se generaron menor aceptación, o desarrollo en su solución, solo dejando enunciadas solo las partes que se desarrollaban de carácter mecánico, de ahí inferimos una ineficiente apropiación del concepto y su respectiva utilidad.

Partiendo de lo anterior se recomienda impartir este concepto con la articulación de diferentes registros de representación semiótica, para formar su asimilación desde diferentes perspectivas, esta premisa encamina el siguiente paso a realizar en la complementación de la ingeniera didáctica presentada en esta investigación, donde se estructurarán y posteriormente se aplicarán de unas secuencias didácticas de función de promover una buena conceptualización en los estudiantes.

\section{Referencias}

Álvarez, D., Colorado, H. \& Ospina, P. (2013). Una propuesta didáctica para la enseñanza del concepto de derivada. Revista Científica, 2, 104110.

Ariza, Á. \& Llinares, S. (2009). Sobre la aplicación y uso del concepto de derivada en el estudio de conceptos económicos en estudiantes de Bachillerato y Universidad. Enseñanza de las Ciencias, 27(1), 121-136.

Badillo Jiménez, E. R., Azcárate, C. \& Font, V. (2011). Análisis de los niveles de comprensión de los objetos f'(a) y f'(x) en profesores de matemáticas. Enseñanza de las Ciencias, 29(2), 0191-206.

Briceño, E., Hernández, J. \& Espino, A. (2018). Análisis de la comprensión de la derivada desde el enfoque gráfico en estudiantes de nivel superior. El Cálculo y su Enseñanza, Enseñanza de las Ciencias y la Matemática, 10, 31-47.

Calderón, D. I. \& Leon, O. L. (2012). Cap. 3 La ingeniería didáctica como metodología de investigación del discurso en el aula. En Lenguaje y Educación: Perspectivas metodológicas y teóricas para su estudio (pp. 71-104). Colombia: Universidad Distrital Francisco José de Caldas. Carabús, O. (2009). El Aprendizaje del Cálculo en la Universidad. La Conceptualización de la Derivada de una Función y sus Niveles de Comprensión. En Congreso regional de ciencia y tecnología (pp. 1-11). Catamarca, Argentina: Universidad Nacional de Catamarca.

Cervantes-Salazar, M., Camarena-Gallardo, P. \& Pinet-Plasencia, R. (2008). La derivada con la matemática en contexto y el enfoque hacia la modelación. Científica, 12(4), 167-173.

Cuevas, C. A., Rodríguez, A., \& González, O. (2014). Introducción al concepto de derivada de una función real con apoyo de las tecnologías digitales. En Lestón, Patricia (Ed.), Acta Latinoamericana de Matemática Educativa (pp. 2335-2345). México, DF: Comité Latinoamericano de Matemática Educativa.

Domínguez-Contreras, V. \& Sanchez-Galeano, M. (2016). El concepto de derivada en estudiantes de educación media. Eco Matemático, 7(1), 8691. https://doi.org/10.22463/17948231.1105

Duval, R. (2006). Un tema crucial en la educación matemática: La habilidad para cambiar el registro de representación. La Gaceta de la Real Sociedad Matemática Española, 9(1), 143-168.

Gallo, A., Manrique, J., Prada, R., (2017). Aplicación de la ingeniería didáctica en el aprendizaje del concepto de función. Eco matemático 8(1). 4348

Gutiérrez, L., Buitrago, M. A. \& Ariza, L. M. (2017). Identificación de dificultades en el aprendizaje del concepto de la derivada y diseño de un OVA como mediación pedagógica. Revista Científica "General José María Córdoba”, 15(20), 137153.

Hashemi, N., Abu, M. S., Kashefi, H. \& Rahimi, K. (2014). Undergraduate students' difficulties in conceptual understanding of derivation. Procedia-Social and Behavioral Sciences, 143, 358-366.

Hernández-Suarez, C. A., Prada-Núñez, R., \& Ramírez-Leal, P. (2017). Obstáculos epistemológicos sobre los conceptos de límite y continuidad en cursos de cálculo diferencial en programas de ingeniería. 
Revista Perspectivas, 2(2), 73-83. https://doi. org/10.22463/25909215.1316

Hitt, F. (2003). Dificultades en el aprendizaje del cálculo 1. En undécimo Encuentro de Profesores de Matemáticas del Nivel Medio Superior (pp. 81-107). Morelia.

Orhun, N. (2012). Graphical understanding in mathematics education: Derivative functions and students' difficulties. Procedia-Social and Behavioral Sciences, 55, 679-684.

Ortega, T. \& Sierra, M. (1998). El concepto de derivada: algunas indicaciones para su enseñanza. Revista interuniversitaria de formación del profesorado, (32), 87-115.

Park, J. (2013). Is the derivative a function? If so, how do students talk about it?. International Journal of Mathematical Education in Science and Technology, 44(5), 624-640. doi:10.1080/0 020739x.2013.795248

Pino, L. R., Godino, J. D. \& Moll, V. F. (2011). Faceta epistémica del conocimiento didácticomatemático sobre la derivada. Educação Matemática Pesquisa: Revista do Programa de Estudos Pós-Graduados em Educação Matemática, 13(1), 141-178.

Pino-Fan, L. R., Godino, J. D. \& Font, V. (2015). Una propuesta para el análisis de las prácticas matemáticas de futuros profesores sobre derivadas. Bolema-Boletim de Educação Matemática, 29(51), 60-89.

Pinto, I., \& Parraguez, M. (2015). El concepto de derivada desde la teoría los modos de pensamiento, sustentada en la epistemología de Cauchy. En Flores, Rebeca (Ed.), Acta Latinoamericana de Matemática Educativa (pp. 337-344). México, DF: Comité Latinoamericano de Matemática Educativa.

Retana, J. Á. G. (2013). La problemática de la enseñanza y el aprendizaje del cálculo para ingeniería. Revista Educación, 37(1), 29-42.

Rodríguez-Pérez, E. (2015). El concepto de derivada y el modelo de Van Hiele en estudiantes de licenciatura en matemáticas e informática de la Universidad. Eco Matemático, 6(1), 43-49. https://doi.org/10.22463/17948231.458

Ruiz, E. F., Gutiérrez, J. J. \& Garay, L. I. (2018). Visualizando problemas de la derivada con aplicaciones en dispositivos móviles. Innovación educativa, 18(76), 39-67.

Salazar. C., Díaz, H. \& Bautista, M. (2009). Descripción de niveles de comprensión del concepto derivada. Tecné Episteme y Didaxis TED, (26), 62-82.

Sánchez-Matamoros-Garcia, G., García Blanco, M. M., \& Llinares Ciscar, S. (2006). El desarrollo del esquema de derivada. Enseñanza de las ciencias. Revista de investigación y experiencias didácticas, 24(1), 85-98.

Santoyo, F., Rangel, M. Á., Santoyo, E. \& Puga, K. L. (2016). Evaluación de una estrategia didáctica para la apropiación del concepto "derivada de una función". RIDE. Revista Iberoamericana para la Investigación y el Desarrollo Educativo, 7(13), 250-272.

Saraza, D. \& Prada, R. (2017). Estado del arte alrededor de la comprensión conceptual de la derivada. En Encuentro Internacional en Educación Matemática (pp. 122-128). Cúcuta, Colombia.

Tall, D. (1993). Students' difficulties in calculus. In proceedings of working group (Vol. 3, pp. 1328).

Vega, M. A., Carrillo, J. \& Soto, J. (2014). Análisis según el modelo cognitivo APOS del aprendizaje construido del concepto de la derivada. Boletim de Educação Matemática, 28(48), 403-429.

Vrancken, S. \& Engler, A. (2014). Una Introducción a la Derivada desde la Variación y el Cambio: resultados de una investigación con estudiantes de primer año de la universidad. Boletim de Educação Matemática, 28(48), 449-468.

Vrancken, S., Engler, A. \& Müller, D. (2010). Una secuencia didáctica para la introducción del concepto de derivada. Resultados de su implementación. En Lestón, Patricia (Ed.), Acta Latinoamericana de Matemática Educativa (pp. 379-388). México, DF: Comité Latinoamericano de Matemática Educativa A. C. 
Weigand, H. G. (2014). A discrete approach to the concept of derivative. ZDM, 46(4), 603-619. 